\title{
Molecular Stripe Patterns on Surfaces in the Presence of Long- Range Repulsive Electrostatic Interactions: Monte Carlo Simulations and Mean-Field Theory
}

\author{
Christoph Schiel, Maximilian Vogtland, Ralf Bechstein, Angelika Kühnle, and Philipp Maass*
}

Cite This: J. Phys. Chem. C 2021, 125, 20650-20657

Read Online

ACCESS | Lلll Metrics \& More 回 Article Recommendations

ABSTRACT: Mobile molecules on surfaces can arrange into stripes due to directional attractive interactions such as $\pi-\pi$ stacking, hydrogen, or covalent bonding. The structural arrangement of the stripes depends on the underlying substrate lattice and omnipresent long-range electrostatic interactions. To model the impact of the interplay of short-range attractive and long-range interactions on the molecular arrangements, we study a coarsegrained theoretical approach, where the attractive interaction is described by an anisotropic Ising model. As for the long-range electrostatic interaction, we focus on repulsive dipole-dipole interactions. An efficient Monte Carlo algorithm is developed by which even stripe patterns with very long stripes can be equilibrated. Using this algorithm, we assess the limits of a

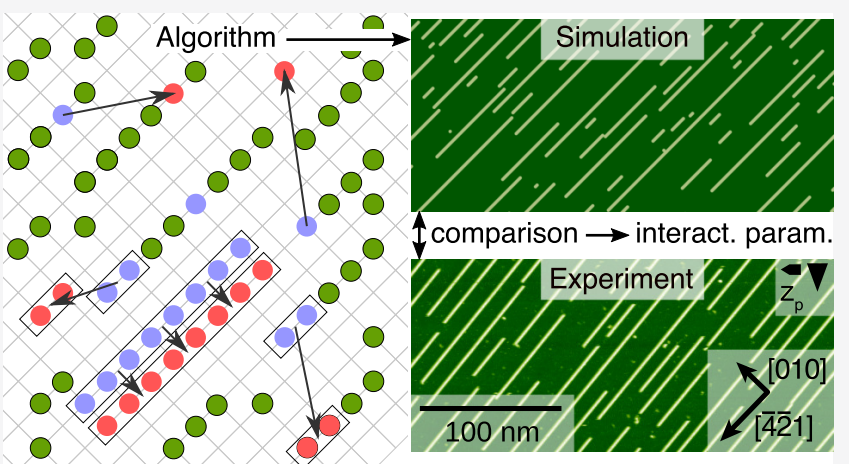
previously developed mean-field theory, which provides analytical predictions for stripe-to-stripe distance and stripe length distributions. This theory allows one to extract interaction parameters by fitting respective distributions to experimental data. We determine the limits of the applicability of the mean-field theory and beyond its limits suggest a combined approach of mean-field analysis and simulations. The power of this approach is demonstrated by applying it to experimental observed stripe pattern of 3hydroxybenzoic acid (3-HBA) on the calcite (10.4) surface.

\section{INTRODUCTION}

Molecular structures on surfaces in the submonolayer regime can be designed with tailored properties because the large variety of functional groups offer many possibilities for steering molecule-molecule and molecule-substrate interactions. ${ }^{1,2}$ In self-assembly of molecules, different types of structures emerge, such as stripes, ordered two-dimensional arrays, irregularly distributed islands, and cross-linked networks. ${ }^{3-9}$

Molecular stripes typically occur due to attractive directional intermolecular interactions mediated by covalent bonding, hydrogen bonding, or $\pi-\pi$ stacking. ${ }^{1}$ Such intermolecular stripe formation mechanisms can be further assisted by a suitable anchoring to the surface. ${ }^{2}$ While these mechanisms have been extensively studied in the past both by experiment and theory, ${ }^{10-12}$ less attention has been paid so far to features of stripe patterns caused by omnipresent electrostatic interactions between charged molecules, or molecules carrying a dipole or higher-order multipole moment. ${ }^{5}$ Multipole moments can be induced also by molecule-molecule or surface-molecule charge transfer. ${ }^{13-16}$ The impact of these long-range interactions should be particularly pronounced on inert surfaces where the molecule-surface interactions are not dominating the structure formation.
To model the stripe formation, we follow a coarse-grained approach here, where the directional attractive interaction is described by a nearest-neighbor interaction. Recently, we developed a mean-field theory ${ }^{17}$ to predict stripe-to-stripe distance and stripe length distributions arising from the interplay between the long-range repulsive and the shortrange attractive interaction. This theory was applied to stripe formation of 3-hydroxybenzoic acid (3-HBA) molecules on the calcite (10.4) surface. $^{5}$ By fitting measured distributions to analytical expressions, we were able to estimate the strength of attractive and repulsive interactions.

It can be expected, however, that the mean-field approach has limits of applicability. For strong attractive and/or repulsive interactions, pronounced fluctuations and correlations in the spatial arrangement of stripes may lead to significant deviations from the mean-field predictions. It is,

Received: July 15, 2021

Published: September 9, 2021 
therefore, necessary to check the mean-field theory against simulations. To apply standard simulation procedures based on moves of single molecules, however, is difficult. Within acceptable computing time, such procedures do not allow one to generate equilibrated patterns of stripes with lengths comparable to that observed in experiments. To overcome this difficulty, we develop an efficient algorithm, which involves moves of whole stripes as well as stripe segments. After introducing the model and parameter specification in Section 2, we describe this algorithm in Section 3. The limits of the mean-field theory obtained by comparison with simulations are described in Section 4. Beyond these limits, one can use the mean-field theory to estimate starting values for the simulations. A combined procedure of mean-field theory and simulations can thus be used, and we demonstrate its power by application to 3-HBA on calcite (10.4).

\section{MODEL AND PARAMETER SPECIFICATION}

We consider a situation where the placement of molecules on a substrate reflects the underlying surface lattice. The lattice is the set of all possible anchoring sites of the molecules, and occupation numbers $n_{i}$ specify whether the site $i$ is occupied $\left(n_{i}\right.$ $=1)$ or not $\left(n_{i}=0\right)$. For simplicity, this lattice is assumed to be rectangular with spacing $a_{\|}$in the direction parallel to the stripes, and with spacing $a_{\perp}$ perpendicular to it. The attractive interaction between molecules is present only in the parallel direction and described by a nearest-neighbor interaction $J$. As for the long-range repulsive interaction, we consider a dipole interaction of strength

$$
\Gamma=\frac{p^{2}}{4 \pi \varepsilon_{0} a_{\|}^{3}}
$$

where $\varepsilon_{0}$ is the electric field constant and $p$ is the dipole moment of the molecules. The energy of the system is given by the lattice gas Hamiltonian

$$
H=-\frac{J}{2} \sum_{i \mathrm{NN} j} n_{i} n_{j}+\frac{\Gamma}{2} \sum_{k, l} \frac{n_{k} n_{l}}{r_{k l}^{3}}
$$

where the sum over $i \mathrm{NN} j$ means the summation over all nearest-neighbor sites in parallel direction. The summations over $k$ and $l$ run over all sites in the lattice, and the dimensionless $r_{k l}$ are the distances between sites $k$ and $l$ in units of $a_{\|}$.

The parameters $J, \Gamma$, and the aspect ratio $a_{\perp} / a_{\|}$are specific for the molecule and the substrate. If these are given, the temperature $T$ and coverage $\theta$ (mean occupation number) can be varied to a certain extent while molecular stripe patterns are forming. As for the interaction parameter $J$, we will study a range $0<\beta J<15$ with $\beta=1 / k_{\mathrm{B}} T$ ( $k_{\mathrm{B}}$ : Boltzmann constant). At room temperature, this corresponds to $0-0.38 \mathrm{eV}$, which covers strengths of hydrogen bonds in organic molecules up to the medium-size range. ${ }^{18}$ As for $\Gamma$ in eq 1 , we consider a length scale $a_{\|} \simeq 1 \mathrm{~nm}$ and $p$ of the order of molecular dipole moments in the range $0-13$ D. ${ }^{19,20}$ The largest dipole moment corresponds to $\beta \Gamma \simeq 4$ at room temperature.

In our previous modeling of the stripe formation of 3-HBA on calcite (10.4), we employed our mean-field theory to estimate the interaction parameters $J$ and $\Gamma$ from distributions $\Psi(l)$ of stripe lengths $l$ and distributions $\Phi(d)$ of stripe-tostripe distances $d$. These distances $d$ are defined as the nearestneighbor spacings of stripes in perpendicular direction, where the nearest-neighbor condition includes the requirement that the respective stripes should overlap after a translation in the perpendicular direction. We found $\beta J \approx 12$ and $\beta \Gamma \approx 2$, which lie in the ranges considered above.

The details of the mean-field treatment are given in ref 17 . Here, we list the necessary equations needed for the analysis: The predicted stripe-to-stripe distance distribution is

$$
\Phi_{\mathrm{mf}}(d)=\frac{1}{Z} \exp (-\beta[f d+U(d)])
$$

where $Z=\sum_{d=1}^{\infty} \exp (-\beta[f d+U(d)])$ and $f$ is fixed by requiring the mean distance

$$
\bar{d}=\sum_{d=1}^{\infty} \mathrm{d} \Phi_{\mathrm{mf}}(d)
$$

to agree with the measured one. The function

$$
U(d)=\frac{p^{2}}{2 \pi \epsilon_{0} d a_{\|}^{2}}\left[\left(1+\frac{\bar{l}^{2}}{d^{2}}\right)^{1 / 2}-1\right]
$$

is the interaction between two parallel stripes, which are at distance $d$ and both have the mean stripe length $\bar{l}$. The predicted stripe length distribution is

$$
\Psi_{\mathrm{mf}}^{\text {th }}(l)=\frac{\theta-C\left(J_{\text {eff }}\right)}{C\left(J_{\text {eff }}\right)}\left(\frac{C\left(J_{\text {eff }}\right)}{\theta}\right)^{l}
$$

with

$$
J_{\text {eff }}=J-\zeta(3) \Gamma
$$

where $\zeta($.$) is the Riemann zeta function, \zeta(3) \cong 1.20206$, and

$$
C(J)=\theta-\frac{\sqrt{1+4 \theta(1-\theta)\left(\mathrm{e}^{\beta J}-1\right)}-1}{2\left(\mathrm{e}^{\beta J}-1\right)}
$$

For estimating the interaction parameters, the tails of experimentally determined distributions $\Phi(d)$ and $\Psi(l)$ are fitted to the behavior predicted by eqs 3 and 6 . In the tail regimes, microscopic details at small length scales should become negligible such that we can expect the description based on the Hamiltonian in eq 2 to become reliable. In practice, we first determine the average stripe length $\bar{l}$ and stripe distance $\bar{d}$. Then, we determine $\Gamma$ by fitting $\Phi(d)$ and thereafter $J$ by fitting $\Psi(l)$.

The limits of validity and the accuracy of the mean-field approach have not yet been evaluated. To this end, simulations must be performed, which is a challenging task because standard Monte Carlo algorithms with moves of single molecules fail to generate equilibrated stripe patterns in reasonable computing time. In the interesting regime, where stripes with lengths much larger than the lattice spacing form, the probability of rearranging long stripe sections by moves of single molecules is very low. What is needed is an efficient algorithm which allows one to move whole stripe segments in the simulation procedure. We develop a corresponding algorithm in Section 3.

Using this simulation procedure, we can generate stripe patterns in a reasonable computing time. For example, typical patterns of 3-HBA molecules on calcite (10.4) observed in the experiment $^{17}$ (see Figure 1a) can be well reproduced (see Figure $1 \mathrm{~b}$ ). The interaction parameters used in the simulation were the ones estimated from the mean-field treatment. We 

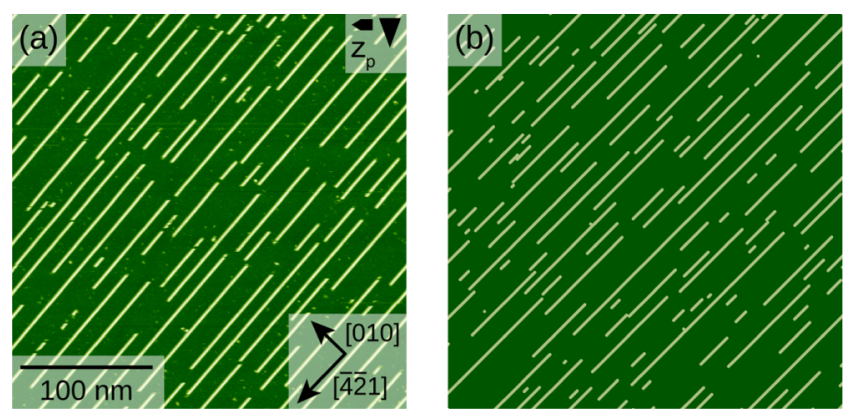

Figure 1. (a) Section of an atomic force microscopy (AFM) topography $\left(z_{\mathrm{p}}\right)$ image of 3-hydroxybenzoic acid (3-HBA) molecules on the calcite surface (10.4) at temperature $290 \mathrm{~K}$ and coverage $\theta=$ 0.11 (complete image has $5 \times 10^{3}$ stripes). (b) Stripe pattern generated based on the Hamiltonian in eq 2 with interaction parameters $J=12.3$ and $\Gamma=1.85$ (in units of thermal energy) for the same coverage and temperature as in the experiment (the size of the pattern corresponds to about $10^{5}$ anchoring sites of 3-HBA dimers).

now can check also how good these estimates are. More generally, we evaluate in Section 4 the regime of interaction parameters where the mean-field theory gives reasonable results. Outside this regime, the mean-field theory is still useful to provide starting values for the interaction parameters, which subsequently are optimized by the simulations. This combined method provides a powerful theoretical tool to analyze the selfassembly of molecules into stripe patterns on surfaces.

\section{SIMULATION PROCEDURE}

The specific algorithm to perform Monte Carlo simulations for the Hamiltonian in eq 2 applies to the canonical ensemble and grounds on known general concepts ${ }^{21-23}$ for factorizing transition probabilities in attempt and acceptance probabilities. In presenting this algorithm, we will speak about particles that can represent different types of molecules. The rectangular lattice introduced above is used for discussing the algorithm. Other types of lattices can be treated similarly.

The states $s$ of the system are the possible sets of the occupation numbers, $s=\left\{n_{i}\right\}$. We introduce three distinct classes of state changes, where (1) single particles, (2) complete stripes, and (3) stripe sections are moved. State changes of classes 2 and 3 are introduced to overcome the otherwise low probability of moving long stripes. The class $i$ is selected with probability $\chi_{i}\left(\sum_{i=1}^{3} \chi_{i}=1\right)$.

In each class, elementary moves are attempted with a probability $g\left(s^{\prime} \mid s\right)$ of changing state $s$ to $s^{\prime}$. A transition $s \rightarrow s^{\prime}$ is accepted with the acceptance probability $a\left(s^{\prime} \mid s\right)$. Certain state changes $s \rightarrow s^{\prime}$ are attempted but always rejected (see below) and accordingly have an acceptance probability $a\left(s^{\prime} \mid s\right)=0$. The respective reverse state changes are not attempted in those cases, i.e., $g\left(s \mid s^{\prime}\right)=0$ [and $a\left(s \mid s^{\prime}\right)$ is irrelevant]. For a transition to be realized, it has to be attempted and accepted. Accordingly, the transition probabilities are given by $w\left(s^{\prime} \mid s\right)$ $=g\left(s^{\prime} \mid s\right) a\left(s^{\prime} \mid s\right)$.

These transition probabilities are required to fulfill the detailed balance condition $p_{\mathrm{eq}}(s) w\left(s^{\prime} \mid s\right)=p_{\mathrm{eq}}\left(s^{\prime}\right) w\left(s \mid s^{\prime}\right)$ to ensure that the equilibrium Boltzmann distribution $p_{\text {eq }}(s) \propto$ $\exp \left[-H(s) / k_{\mathrm{B}} T\right]$ is approached. This can be achieved by an appropriate choice of the acceptance probabilities. We here choose the Metropolis form

$$
a\left(s^{\prime} \mid s\right)=\min \left(1, \frac{p_{\mathrm{eq}}\left(s^{\prime}\right) g\left(s \mid s^{\prime}\right)}{p_{\mathrm{eq}}(s) g\left(s^{\prime} \mid s\right)}\right)
$$

Note that $g\left(s \mid s^{\prime}\right) / g\left(s^{\prime} \mid s\right)$ is the ratio between the attempt probabilities for a transition $s \rightarrow s^{\prime}$ and its reverse if $a\left(s^{\prime} \mid s\right) \neq 0$. [For $g\left(s^{\prime} \mid s\right) \neq 0$ and $g\left(s \mid s^{\prime}\right)=0$ (reverse transition not attempted), $a\left(s^{\prime} \mid s\right)=0$ (transition is rejected).]

We now describe the elementary trial moves in classes 1 to 3 , where those belonging to classes 2 and 3 are furthermore illustrated in Figure 2. When referring to a "random selection", we mean a selection with equal probability among units whose specification becomes clear from the context.

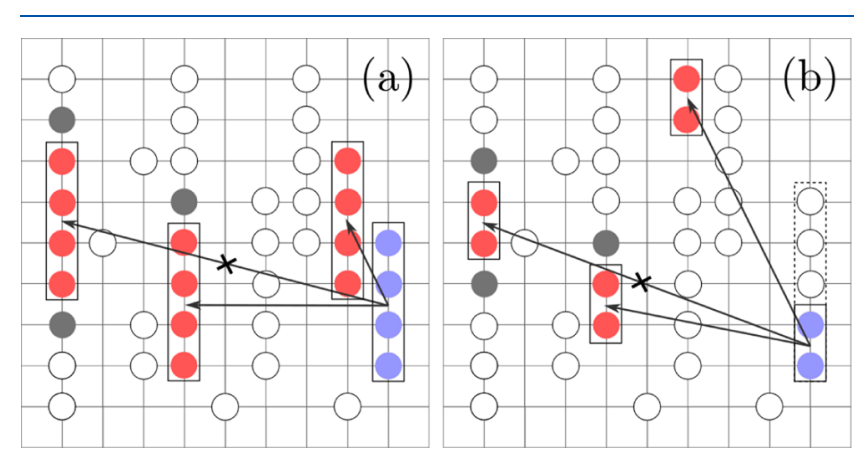

Figure 2. Possible moves (a) in class 2 , where whole stripes are displaced, and (b) in class 3 , where section of stripes are moved to another position. The particles marked in blue are at the initial position, and the particles marked in red are at a new position after a move. At their new positions, the displaced stripes in (a) and displaced stripe sections in (b) have zero, one, or two occupied particles as nearest neighbors at their ends, which are marked in gray. In the cases of two occupied nearest neighbors, the moves are rejected as indicated by the crossing out of the respective arrows.

Class 1: One particle is randomly picked and moved to another site randomly selected from all vacant sites. Note that the particle in its initial state can be isolated or part of a stripe.

Class 2: One complete stripe with length $l \geq 2$ is randomly picked and moved to another randomly chosen position. The restriction $l \geq 2$ excludes single-particle moves already considered in class 1 . If the stripe at the new position overlaps with at least one particle, the move is rejected. The move is also rejected if both end members of the stripe in the target state have nearest neighbors. This would imply that the stripe is closing a gap between two other stripes, as indicated by the crossed-out move attempt in Figure 2a. As we do not allow for the reverse process in such a case, the rejection is required to fulfill the detailed balance condition.

Class 3: One stripe of length $l \geq 3$ together with one of its ends is randomly selected. The restriction $l \geq 3$ excludes moves already considered in classes 1 and 2 . A section of random length $k \in\{2, \ldots, l-1\}$ from the selected stripe end is then taken and moved to another position. As for the moves in class 2 , the move is rejected if it leads to an overlap with another particle or to a gap-filling between stripes.

The ratio $g\left(s \mid s^{\prime}\right) / g\left(s^{\prime} \mid s\right)$ in eq 9 is always equal to 1 except for three cases which need special consideration. To specify the respective ratios for these three cases, we first introduce two further probabilities in a state $s$ : the probability $\gamma_{2}(s)$ of selecting a certain stripe, and the probability $\gamma_{3}(s, l)$ of picking a certain stripe with length $l \geq 3$ and a section $k \in\{2, \ldots, l-2\}$ from this stripe. The probability $\gamma_{2}(s)$ is given by 


$$
\gamma_{2}(s)=\frac{1}{N_{\geq 2}(s)}
$$

where $N_{\geq 2}(s)\left[N_{\geq j}(s)\right]$ is the number of stripes with length $l \geq$ $2[l \geq j]$. The probability $\gamma_{3}(s)$ is equal to $1 / N_{\geq 3}(s)$ (random selection of stripe with length $l \geq 3$ ) times $1 / 2$ (a section of length $k \in\{2, \ldots, l-2\}$ is chosen from either one of the stripe ends) times $1 /(l-2)$ (there are $(l-2)$ possibilities for $k$ )

$$
\gamma_{3}(s, l)=\frac{1}{2(l-2) N_{\geq 3}(s)}
$$

Having specified $\gamma_{2}(s)$ and $\gamma_{3}(s, l)$, the three state changes $s \rightarrow$ $s^{\prime}$ with $g\left(s \mid s^{\prime}\right) / g\left(s^{\prime} \mid s\right) \neq 1$ are:

(i) If a stripe with length $l_{1} \geq 2$ is attempted to move and to be attached to another stripe with length $l_{2} \geq 2$ [class 2 move for $s \rightarrow s^{\prime}$ and class 3 move for $s^{\prime} \rightarrow s$ ], it holds

$$
\begin{aligned}
\frac{g\left(s \mid s^{\prime}\right)}{g\left(s^{\prime} \mid s\right)} & =\frac{\chi_{3} \gamma_{3}\left(s^{\prime}, l_{1}+l_{2}\right)}{\chi_{2} \gamma_{2}(s)} \\
& =\frac{\chi_{3}}{\chi_{2}} \frac{N_{\geq 2}(s)}{2\left(l_{1}+l_{2}-2\right) N_{\geq 3}\left(s^{\prime}\right)}
\end{aligned}
$$

(ii) If a section of length $k \in\{2, \ldots, l-2\}$ from a stripe with length $l \geq 3$ is moved to a free position where it becomes a new stripe of length $k$ [class 3 move for $s \rightarrow s^{\prime}$ and class 2 move for $s^{\prime} \rightarrow s$ ], it holds

$$
\frac{g\left(s \mid s^{\prime}\right)}{g\left(s^{\prime} \mid s\right)}=\frac{\chi_{2} \gamma_{2}\left(s^{\prime}\right)}{\chi_{3} \gamma_{3}(s, l)}=\frac{\chi_{2}}{\chi_{3}} \frac{2(l-2) N_{\geq 3}(s)}{N_{\geq 2}\left(s^{\prime}\right)}
$$

(iii) If a section of length $k \in\left\{2, \ldots, l_{1}-2\right\}$ from a stripe with length $l_{1} \geq 3$ is moved and attached to another stripe (or single particle) with length $l_{2} \geq 1$ [class 3 move for both $s \rightarrow s^{\prime}$ and $\left.s^{\prime} \rightarrow s\right]$, it holds

$$
\frac{g\left(s \mid s^{\prime}\right)}{g\left(s^{\prime} \mid s\right)}=\frac{\gamma_{3}\left(s^{\prime}, k+l_{2}\right)}{\gamma_{3}\left(s, l_{1}\right)}=\frac{\left(l_{1}-2\right) N_{\geq 3}(s)}{\left(k+l_{2}-2\right) N_{\geq 3}\left(s^{\prime}\right)}
$$

For the moves in classes $1-3$ as well as the interaction between particles, we applied periodic boundary conditions, and in all simulations, we chose $\chi_{i}=1 / 3$.

Representative equilibrated stripe patterns for varying interaction parameters $J$ and $\Gamma$ generated with the algorithm are shown in Figures 3 and 4. When decreasing $J$ and/or increasing $\Gamma$, the stripes become shorter until essentially only single particles are left and one can no longer speak about a stripe pattern. The dipole-dipole interaction was calculated using the minimum image convention. ${ }^{22}$

To control the equilibration, we have checked that the system's energy, mean stripe-to-stripe distance and mean stripe length, as well as the second- and third-order cumulants of the stripe-to-stripe distance and stripe length distributions were fluctuating around constant values. We also checked that our findings were the same for different starting configurations.

\section{DETERMINING INTERACTION PARAMETERS FROM STRIPE PATTERNS}

4.1. Limits of the Mean-Field Theory. With the efficient algorithm presented in Section 3, we are now able to assess the accuracy of the mean-field theory and to determine its limits.
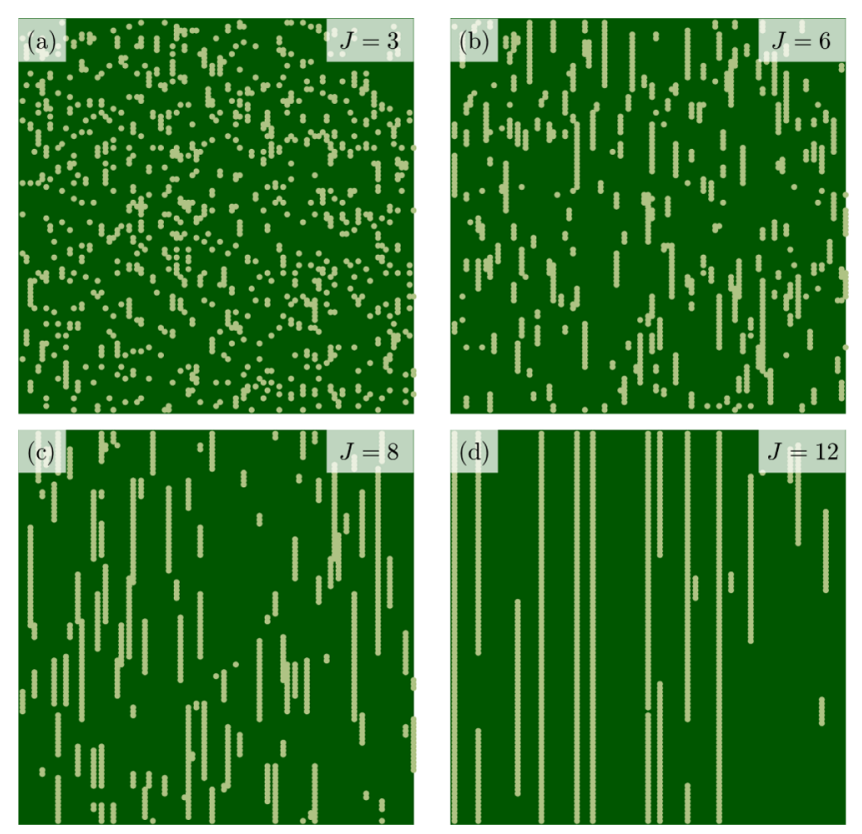

Figure 3. Equilibrated stripe patterns for fixed $\Gamma=1$ and four different values of (a) $J=3$, (b) $J=6$, (c) $J=8$, and (d) $J=12$ (all energies in units of $\left.k_{\mathrm{B}} T\right)$. The simulations were performed for a coverage of $\theta=$ 0.1 and a lattice of size $N_{\perp} \times N_{\|}=100 \times 100$ with an aspect ratio of lattice constants $a_{\perp} / a_{\|}=2.5$.
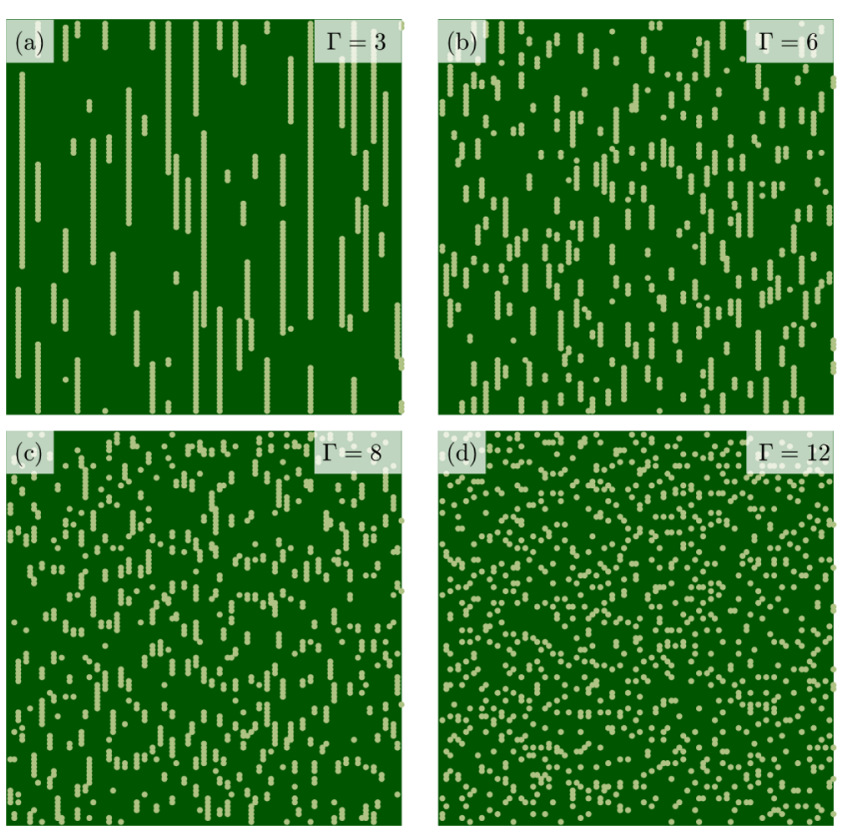

Figure 4. Equilibrated stripe patterns for fixed $J=1$ and four different values of (a) $\Gamma=3$, (b) $\Gamma=6$, (c) $\Gamma=8$, and (d) $\Gamma=12$ (all energies in units of $\left.k_{\mathrm{B}} T\right)$. Other simulation parameters are as in Figure 3.

To that end, we will apply the mean-field approach to distributions $\Phi(d)$ and $\Psi(l)$ generated from simulations. We focus here on the modeling of stripe patterns formed by 3HBA molecules on calcite (10.4) around a coverage $\theta=0.1$, where experimental data have been measured. These stripes consist of rows of 3-HBA dimers with a width of $2 \mathrm{~nm}$ and a periodicity of $0.8 \mathrm{~nm}$ in stripe direction. The dimers are treated as particles in our algorithm and occupy anchoring sites of a 
rectangular lattice with an aspect ratio $a_{\perp} / a_{\|}=2 \mathrm{~nm} / 0.8 \mathrm{~nm}=$ 2.5 .

As the stripe distances are short while the stripes can become very long, we chose an asymmetric simulation geometry with a total number of $N_{\perp} \times N_{\|}=100 \times 2500$ anchoring sites. We found this size to be sufficient for our results to be unaffected by finite size effects for the parameter regimes $0<J<15$ and $0<\Gamma<4$, where here and in the following we use $k_{\mathrm{B}} T$ as the energy unit. The coverage is $\theta=$ 0.1 for all results referring to the evaluation of the mean-field theory. When modeling distributions sampled from the experiments in Sections 4.2 and 4.3, the simulations are carried out for the coverage $\theta=0.11$ of the experiment.

Figure 5 shows the simulated $\Phi(d)$ and $\Psi(l)$ (gray bars) for $J=12.3$ and $\Gamma=1.85$, which were found before as estimates

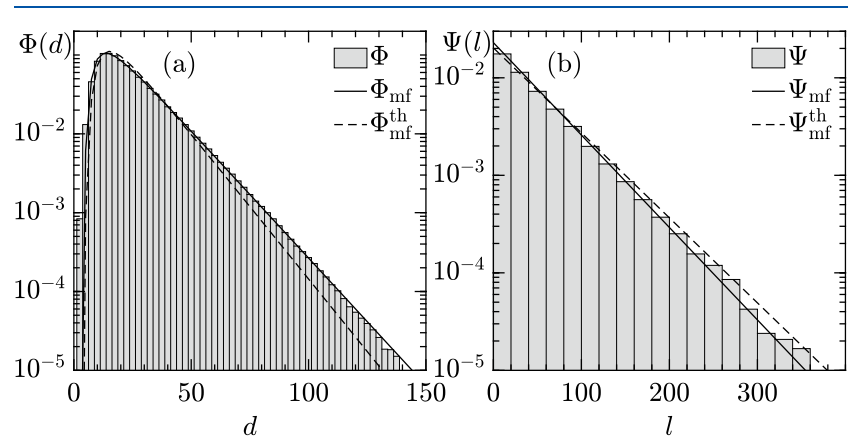

Figure 5. Simulated results for (a) the distribution $\Phi(d)$ of stripe-tostripe distances and (b) the distribution $\Psi(l)$ of stripe lengths at a coverage $\theta=0.1$, and interaction parameters $J=12.3$ and $\Gamma=1.85$ (in units of $k_{\mathrm{B}} T$ ). The dashed black lines mark the theoretical mean-field predictions for $\Phi_{\mathrm{mf}}^{\mathrm{th}}(d)$ and $\Psi_{\mathrm{mf}}^{\mathrm{th}}(l)$ according to eqs 3 and 6 . The solid black lines indicate $\Phi_{\mathrm{mf}}(d)$ and $\Psi_{\mathrm{mf}}(l)$ for interaction parameters $J_{\mathrm{mf}}=$ 11.26 and $\Gamma_{\mathrm{mf}}=1.17$ that are obtained by fitting the tails of the simulated distributions (see text). The simulation was carried out for a lattice of $N_{\perp} \times N_{\|}=100 \times 2500$ sites with an aspect ratio of lattice constants $a_{\perp} / a_{\|}=2.5$.

from applying the mean-field theory to the experiment (see also Figure 1). The dashed lines refer to the theoretical meanfield distributions $\Phi_{\mathrm{mf}}^{\text {th }}(d)$ and $\Psi_{\mathrm{mf}}^{\text {th }}(l)$ from eqs 3 and 6 , if the interaction parameters were already known. As can be seen in Figure $5 b$, the predicted stripe length distribution is close to the simulated one, while pronounced differences can be seen between $\Phi_{\mathrm{mf}}^{\text {th }}(d)$ and $\Phi(d)$ in Figure 5a.

Of course, when applying the mean-field theory, $J$ and $\Gamma$ are not known and estimates $J_{\mathrm{mf}}$ and $\Gamma_{\mathrm{mf}}$ must be determined by applying the tail-fitting procedure described in Section 2 . When looking at the simulated stripe-to-stripe distance distribution in Figure 5a, we consider the tail region to start at a distance $10 \%$ larger than that $d$, where $\Phi(d)$ has its maximum. The best fit to $\ln \Phi(d)$ according to the method of least-squares gives our mean-field estimate $\Gamma_{\mathrm{mf}}$. The stripe length distribution $\Psi(l)$ in Figure 5 b can be fitted to eq 6 over the full range of $l$ values. The asymptotic decay $\Psi_{\mathrm{mf}}(l) \sim \mathrm{e}^{-l / l_{0}}$, $l_{0}=a_{\|} / \ln \left(\theta / C\left(J_{\text {eff }}\right)\right)[$ see eq $(6)]$, gives $J_{\text {eff }}=J_{\mathrm{mf}}-\zeta(3) \Gamma_{\mathrm{mf}}$ and hence $J_{\mathrm{mf}}$.

We obtain $J_{\mathrm{mf}} \simeq 11.26$ and $\Gamma_{\mathrm{mf}} \simeq 1.17$ as estimates. The corresponding distributions are indicated by the solid lines in Figure 5a,b. The estimated parameter $J_{\mathrm{mf}}$ is close to the true value $J=12.3$, while $\Gamma_{\mathrm{mf}}$ underestimates $\Gamma=1.85$. To conclude, the mean-field theory for $J=12.3$ and $\Gamma=1.85$ can be used to obtain a first rough estimate for the interaction parameters, but additional simulations should be carried out to determine more accurate values.

To assess the mean-field approach more generally, we have performed a large number of simulations in a wide parameter regime of $J$ and $\Gamma$, and determined $J_{\mathrm{mf}}$ and $\Gamma_{\mathrm{mf}}$ in the same way as in Figure 5. Our results for $\Gamma=1$ and $\Gamma=2$ and various $J$ in the range 1-14 are shown in Figure 6. As expected, we observe
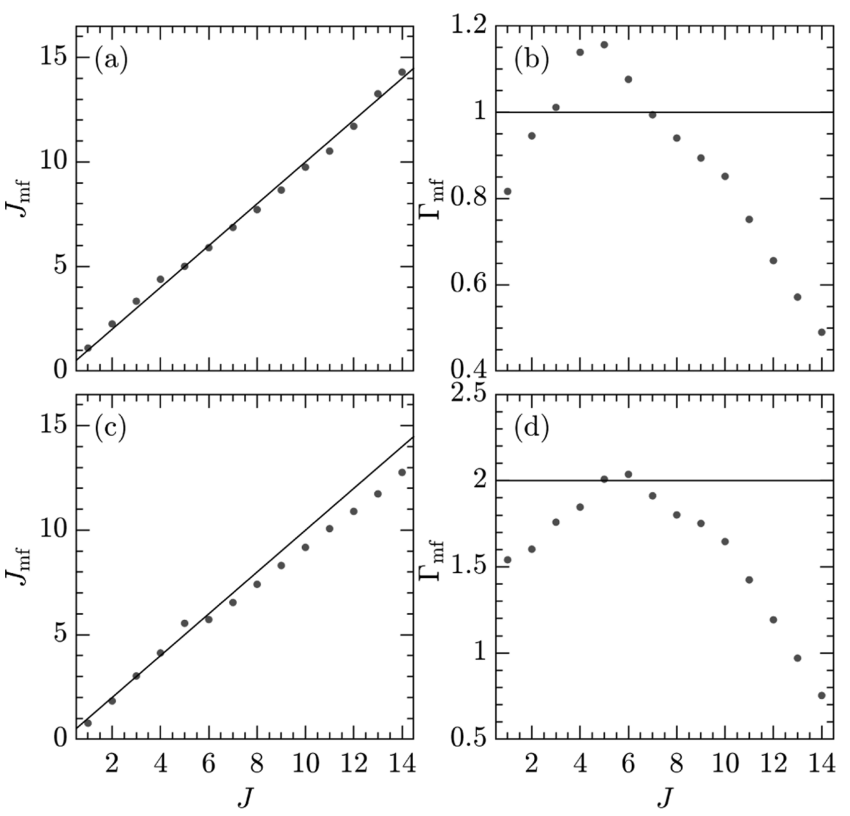

Figure 6. Interaction parameters $J_{\mathrm{mf}}$ and $\Gamma_{\mathrm{mf}}$ (circles) extracted by applying the mean-field theory to the simulated distributions in comparison with the input parameters $J$ and $\Gamma$ of the simulations (indicated by the black lines). In (a) and (b), $J_{\mathrm{mf}}$ and $\Gamma_{\mathrm{mf}}$ are displayed as a function of $J$ for $\Gamma=1$, respectively. In (c) and (d), $J_{\mathrm{mf}}$ and $\Gamma_{\mathrm{mf}}$ are shown for $\Gamma=2$. All parameters are given in units of $k_{\mathrm{B}} T$.

larger deviations at higher interaction parameters. Note that the regime $\Gamma>J$ is not of interest as there is no stripe formation (mean stripe length becomes of the order of 1 ).

For $\Gamma=1, J_{\mathrm{mf}}$ is close to $J$. At larger $\Gamma \gtrsim 2$, we find $J_{\mathrm{mf}}<J$ for large $J$, see Figure $6 \mathrm{c}$. The $J_{\mathrm{mf}}$ values are accurate only in a limited interval for small $J$, with the interval narrowing with increasing $\Gamma$. As for the $\Gamma_{\mathrm{mf}}$ values, these are less well predicted by the mean-field theory. As a rule, based on a $20 \%$ deviation, $\Gamma_{\text {mf }}$ yields a reasonable estimate in the parameter range $J=2-$ 10 and $\Gamma \lesssim 2$.

4.2. Improvement of Interaction Parameter Estimates by Simulations. Beyond the limits of the mean-field theory, simulations should be performed with the algorithm described in Section 3, with the mean-field estimates $J_{\mathrm{mf}}$ and $\Gamma_{\mathrm{mf}}$ as starting values. This combined approach is now demonstrated for the experiment based on the previously measured data of 3HBA on calcite (10.4). ${ }^{17}$

We performed simulations in the regimes bounded by $J_{\mathrm{mf}} \pm$ 4 and $\Gamma_{\mathrm{mf}} \pm 2$ and compared the asymptotic decays of the simulated distributions $\Phi_{\text {sim }}(d)$ and $\Psi_{\text {sim }}(l)$ with the decays of the experimental distributions $\Phi(d)$ and $\Psi(l)$. Looking at these experimental distributions in Figure 7 , the tail regions were considered to start at $d=17.5$ and $l=50$. A best fit to an exponential decay in Figure $7 \mathrm{~b}$ gives a decay length $l_{0}=70$. This value, however, needs to be considered with caution because the statistical accuracy for $\Psi(l)$ is limited as the stripes are rather long (see Figure 1) and thus relatively small in 

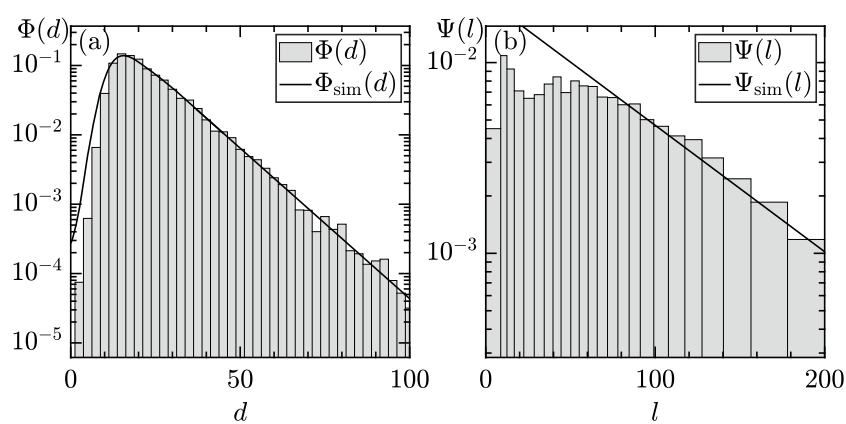

Figure 7. (a) Stripe-to-stripe distance distribution $\Phi(d)$ and (b) stripe length distribution $\Psi(l)$ for stripe patterns formed by 3-HBA molecules on the calcite (10.4) surface at temperature $290 \mathrm{~K}$ and coverage $\theta=0.11$ [see Figure 1a]. The solid lines refer to simulated distributions of equilibrated stripe patterns based on the Hamiltonian in eq 2 for the optimal interaction parameters $J=14.8$ and $\Gamma=3.4$ (in units of $k_{\mathrm{B}} T$ ). The simulated distribution in (b) was renormalized to match the asymptotic decay of $\Psi(l)$. For obtaining $\Psi(l)$, bins of varying size were used with approximately equal amounts of sampled stripe lengths in each bin.

number. In total, $5 \times 10^{3}$ stripe lengths were sampled from the AFM image, which is much smaller than the total number of 4 $\times 10^{5}$ of sampled stripe distances $d$. In view of this, we apply the following procedure for finding the optimal pair of $J$ and $\Gamma$ values:

$R^{2}$ values (coefficient of determination) were calculated with respect to the residuals $\left[\ln \Phi(d)-\ln \Phi_{\text {sim }}(d)\right]$, corresponding to the representation in Figure $5 \mathrm{~b}$ on a semilogarithmic scale. The optimal pair of interaction parameters is the one with the highest $R^{2}$ value under the constraint that the decay length $l_{0}^{\text {sim }}$ of $\Psi_{\text {sim }}(l)$ does not deviate by more than $15 \%$ from $l_{0}$. This gives $\Gamma=3.4$ (yielding $R^{2}=0.989$ ) and $J=14.8$. The corresponding $\Phi_{\text {sim }}(d)$ and $\Psi_{\text {sim }}(l)$ are shown in Figure 7 as solid lines. We note that the data of $\Psi_{\text {sim }}(l)$ were renormalized to match the asymptotic decay of $\Psi(l)$ and the length distributions were determined using bins of varying size with approximately equal amounts of events in each bin.

Our simulations hence yield the more accurate estimates $J=$ 14.8 and $\Gamma=3.4$ in comparison to the values $J_{\mathrm{mf}}=12.3$ and $\Gamma_{\mathrm{mf}}$ $=1.85$ formerly predicted by applying the mean-field theory only. For the estimated dipole moment, we obtain the modified value $p=8.4 \mathrm{D}$ (former value was $p=6.1 \mathrm{D}$ ).

4.3. Distributions of Stripe Distances between Second and Third Nearest-Neighbor Stripes. The procedure of determining optimal values of $J$ and $\Gamma$ from the simulations could be refined by a more detailed analysis of the information contained in the stripe distances in the perpendicular direction, where the experimental data exhibit higher statistical accuracy. We tried to include information on the distribution of distances between stripes beyond nearestneighbor ones.

For this purpose, we investigated the distribution $\Phi_{2}(d)$ of distances between second nearest-neighbor stripes (orthogonal to the stripe direction). In the absence of correlations, i.e., when successive distances between stripes are independent of each other, this distribution is given by the convolution $\Phi_{2}^{0}=$ $\Phi * \Phi$. This means that we can quantify correlation effects by the differences $\left[\Phi_{2}(d)-\Phi_{2}^{0}\right]$, where $\Phi_{2}(d)$ is the experimentally observed distribution for the second nearestneighbor distances.
Surprisingly, as shown in Figure 8a, no significant differences can be found between $\Phi_{2}(d)$ and $\Phi_{2}^{0}$. This implies that we

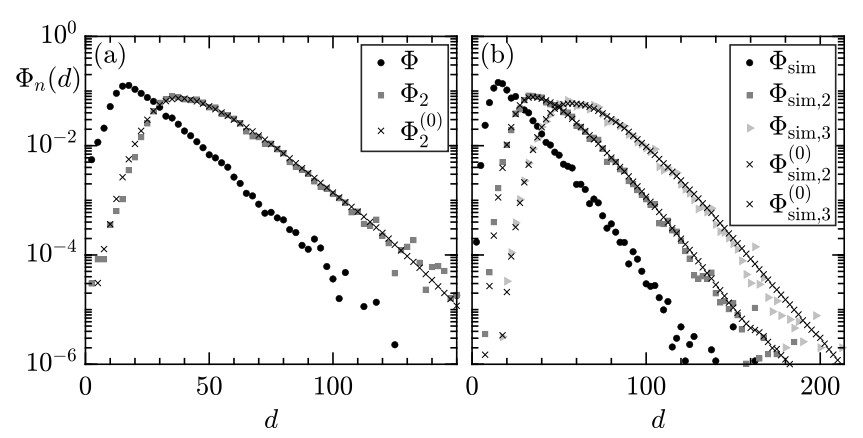

Figure 8. (a) Stripe-to-stripe distance distribution $\Phi(d)$ from Figure $7 \mathrm{a}$ and distribution $\Phi_{2}(d)$ of distances between second nearestneighbor 3-HBA stripes in comparison with $\Phi_{2}^{0}(d)=(\Phi * \Phi)(d)$. (b) Simulated stripe-to-stripe distance distribution $\Phi_{\text {sim }}(d)$ from Figure $7 \mathrm{a}$, and second and third nearest-neighbor distance distributions $\Phi_{\mathrm{sim}, 2}(d)$ and $\Phi_{\mathrm{sim}, 3}(d)$ in comparison with $\Phi_{\mathrm{sim}, 2}^{0}(d)$ and $\Phi_{\mathrm{sim}, 3}^{0}(d)$ obtained by convolving $\Phi_{\text {sim }}(d)$.

cannot utilize information on stripe distances beyond nearest neighbors to improve our procedure for estimating $J$ and $\Gamma$ in the present case. The fact that corresponding correlation effects are negligible is confirmed by our simulations; see Figure $8 b$, where we furthermore compare simulated results for the distribution $\Phi_{3}$ of third nearest-neighbor stripe distances with $\Phi_{3}^{0}=\Phi^{*} \Phi^{*} \Phi$.

\section{CONCLUSIONS}

In summary, we have studied the formation of stripe patterns based on a coarse-grained lattice model with competing shortrange attractive and long-range repulsive interactions between the molecules. The short-range attractive interaction was described by an anisotropic Ising model, which can account effectively for covalent bonding, hydrogen bonding, or $\pi-\pi$ stacking. As for the long-range repulsive interactions, we focused on dipole-dipole interactions as a typical case of the omnipresent electrostatic interactions.

We presented a Monte Carlo algorithm by which equilibrated stripe patterns could be generated in reasonable computing time for even very long stripes composed of $10^{3}$ molecular units and more. Using this algorithm, we evaluated a previously developed mean-field theory, ${ }^{17}$ which allows one to determine interaction parameters from stripe-to-stripe distance and stripe length distributions $\Phi(d)$ and $\Psi(l)$, respectively. It turned out that the predictive power of the mean-field theory is limited to weak dipole-dipole interactions (dipole moment not larger than about $\simeq 6 \mathrm{D}$ ) and weak to moderate shortrange attractive interaction. To improve the accuracy, we proposed a combined procedure of mean-field theory and Monte Carlo simulation and applied it to reevaluate experimental data for 3-HBA molecules forming stripes on the calcite (10.4) surface.

As for the coverage $\theta$ of the substrate by the molecules, we have concentrated here on $\theta=0.1$, which is a typical value where stripe formation is seen in experiments. Generally, the range of coverage is limited as for small $\theta$ only single particles and very short chains appear, while at high $\theta$, coalescence events and/or island growth in the direction perpendicular to the substrate become relevant. Our combined procedure, of 
course, is not limited to particular values of the coverage in the regime of stripe formation.

There is also no restriction regarding the lattice symmetry. We here investigated a rectangular lattice with a specific ratio $a_{\|} / a_{\perp}$ of lattice constants in and perpendicular to the stripe direction. This choice was taken to apply the procedure to the available data for stripe formation of 3-HBA molecules on calcite (10.4). The algorithm can be straightforwardly modified or extended to simulate other lattice symmetries.

Improvements of the analysis are possible by including other information than provided by $\Phi(d)$ and $\Psi(l)$. In practice, experimental information on quantities sensitive to the perpendicular direction of stripe formation will be accessible with much higher statistical accuracy. This is because the number of distances in the perpendicular direction is typically much larger than in the parallel direction (if the mean stripe distance is much smaller than the mean stripe length). The stripe pattern in Figure 1 represents an example of this typical situation.

We suggest to examine also the distributions between second and third nearest-neighbor stripe distances in the perpendicular direction. In the absence of correlations between successive stripe distances, these distributions of distances between second (or higher)-order nearest neighbors are given by convolutions of $\Phi(d)$. Accordingly, correlation effects can be identified by comparison with the respective convolutions. For the stripe formation of 3-HBA molecules on the calcite (10.4) surface, we could not identify any such correlations, in agreement also with our simulated data. Accordingly, we could not improve the accuracy of our analysis for this system. This does not preclude that a corresponding analysis would not be informative for other systems of stripe-forming molecules on surfaces. In the case of stripe formation of 3-HBA molecules on calcite, our results show that fluctuation effects must be the primary cause for the differences found between mean-field theory and simulations.

In our modeling, we have assumed a constant dipole moment of the 3-HBA molecules. Alternatively, the dipole moment could arise from a molecular polarizability. The strength of the dipole moment of each molecule would then depend on its local environment, i.e., where it is located within a stripe and how stripes in the environment are positioned. An approach with a molecular polarizability could also be successful in modeling the experimentally observed stripe-tostripe distance and stripe length distributions. The question whether a constant dipole is present could be answered by checking if the experimental findings for other coverages and temperatures can be described by the same values of the dipole moment $p$ and attractive interaction $J$ (given in temperatureindependent units).

\section{AUTHOR INFORMATION}

\section{Corresponding Author}

Philipp Maass - Universität Osnabrück, Fachbereich Physik, D-49076 Osnabrück, Germany; (1) orcid.org/0000-0002-

1268-8688; Email: maass@uni-osnabrueck.de

\section{Authors \\ Christoph Schiel - Universität Osnabrück, Fachbereich Physik, D-49076 Osnabrück, Germany \\ Maximilian Vogtland - Fakultät für Chemie, Universität Bielefeld, D-33615 Bielefeld, Germany}

Ralf Bechstein - Fakultät für Chemie, Universität Bielefeld, D33615 Bielefeld, Germany

Angelika Kühnle - Fakultät für Chemie, Universität Bielefeld, D-33615 Bielefeld, Germany; (1) orcid.org/0000-00031214-1006

Complete contact information is available at:

https://pubs.acs.org/10.1021/acs.jpcc.1c06305

\section{Notes}

The authors declare no competing financial interest.

\section{REFERENCES}

(1) Barth, J. V. Molecular architectonic on metal surfaces. Annu. Rev. Phys. Chem. 2007, 58, 375-407.

(2) Rahe, P.; Kittelmann, M.; Neff, J. L.; Nimmrich, M.; Reichling, M.; Maass, P.; Kühnle, A. Tuning Molecular Self-Assembly on Bulk Insulator Surfaces by Anchoring of the Organic Building Blocks. Adv. Mater. 2013, 25, 3948-3956.

(3) Barth, J. V.; Costantini, G.; Kern, K. Engineering atomic and molecular nanostructures at surfaces. Nature 2005, 437, 671-679.

(4) Auwärter, W.; Schiffrin, A.; Weber-Bargioni, A.; Pennec, Y.; Riemann, A.; Barth, J. Molecular nanoscience and engineering on surfaces. Int. J. Nanotechnol. 2008, 5, 1171-1193.

(5) Neff, J. L.; Söngen, H.; Bechstein, R.; Maass, P.; Kühnle, A. Long-Range Order Induced by Intrinsic Repulsion on an Insulating Substrate. J. Phys. Chem. C 2015, 119, 24927-24931.

(6) Ardhuin, T.; Guillermet, O.; Gourdon, A.; Gauthier, S. Molecular resonance imaging and manipulation of hexabenzocoronene on $\mathrm{NaCl}(001)$ and $\mathrm{KBr}(001)$ on $\mathrm{Ag}$ (111). J. Phys. Chem. C 2018, 122, 11905-11910.

(7) Olszowski, P.; Zajac, L.; Godlewski, S.; Such, B.; Pawlak, R.; Hinaut, A.; Jöhr, R.; Glatzel, T.; Meyer, E.; Szymonski, M. Ordering of $\mathrm{Zn}$-centered porphyrin and phthalocyanine on TiO2 (011): STM studies. Beilstein J. Nanotechnol. 2017, 8, 99-107.

(8) Zajac, L.; Olszowski, P.; Godlewski, S.; Bodek, L.; Such, B.; Jöhr, R.; Pawlak, R.; Hinaut, A.; Glatzel, T.; Meyer, E.; et al. Self-assembling of $\mathrm{Zn}$ porphyrins on a (110) face of rutile $\mathrm{TiO} 2$ - The anchoring role of carboxyl groups. Appl. Surf. Sci. 2016, 379, 277-281.

(9) Gaberle, J.; Gao, D. Z.; Shluger, A. L.; Amrous, A.; Bocquet, F.; Nony, L.; Para, F.; Loppacher, C.; Lamare, S.; Cherioux, F. Morphology and growth mechanisms of self-assembled films on insulating substrates: role of molecular flexibility and entropy. J. Phys. Chem. C 2017, 121, 4393-4403.

(10) Mali, K. S.; Pearce, N.; De Feyter, S.; Champness, N. R. Frontiers of supramolecular chemistry at solid surfaces. Chem. Soc. Rev. 2017, 46, 2520-2542.

(11) Otero, R.; Gallego, J. M.; de Parga, A. L. V.; Martin, N.; Miranda, R. Molecular Self-Assembly at Solid Surfaces. Adv. Mater. 2011, 23, 5148-5176.

(12) Goronzy, D. P.; Ebrahimi, M.; Rosei, F.; Arramel; Fang, Y.; De Feyter, S.; Tait, S. L.; Wang, C.; Beton, P. H.; Wee, A. T.; et al. Supramolecular assemblies on surfaces: nanopatterning, functionality, and reactivity. ACS Nano 2018, 12, 7445-7481.

(13) Stadler, C.; Hansen, S.; Kröger, I.; Kumpf, C.; Umbach, E. Tuning intermolecular interaction in long-range-ordered submonolayer organic films. Nat. Phys. 2009, 5, 153-158.

(14) Fraxedas, J.; García-Gil, S.; Monturet, S.; Lorente, N.; Fernandez-Torrente, I.; Franke, K.; Pascual, J.; Vollmer, A.; Blum, R.-P.; Koch, N.; et al. Modulation of surface charge transfer through competing long-range repulsive versus short-range attractive interactions. J. Phys. Chem. C 2011, 115, 18640-18648.

(15) Fernandez-Torrente, I.; Monturet, S.; Franke, K.; Fraxedas, J.; Lorente, N.; Pascual, J. Long-range repulsive interaction between molecules on a metal surface induced by charge transfer. Phys. Rev. Lett. 2007, 99, No. 176103. 
(16) Matvija, P.; Rozbořil, F.; Sobotík, P.; Ošt’ádal, I.; Pieczyrak, B.; Jurczyszyn, L.; Kocán, P. Electric-field-controlled phase transition in a 2D molecular layer. Sci. Rep. 2017, 7, No. 7357.

(17) Schiel, C.; Vogtland, M.; Bechstein, R.; Kühnle, A.; Maass, P. Molecular self-assembly: Quantifying the balance between intermolecular attraction and repulsion from distance and length distributions. J. Phys. Chem. C 2020, 124, 21583-21590.

(18) Jeffrey, G. A.; Jeffrey, G. A. An Introduction to Hydrogen Bonding; Oxford University Press, New York, 1997; Vol. 12.

(19) NIST Computational Chemistry Comparison and Benchmark Database, NIST Standard Reference Database Number 101. 2020; Release 21, August 2020, Editor: Russell D. Johnson III, http:// cccbdb.nist.gov/.

(20) Kunkel, D. A.; Hooper, J.; Simpson, S.; Miller, D. P.; Routaboul, L.; Braunstein, P.; Doudin, B.; Beniwal, S.; Dowben, P.; Skomski, R.; et al. Self-assembly of strongly dipolar molecules on metal surfaces. J. Chem. Phys. 2015, 142, No. 101921.

(21) Metropolis, N.; Rosenbluth, A. W.; Rosenbluth, M. N.; Teller, A. H.; Teller, E. Equation of state calculations by fast computing machines. J. Chem. Phys. 1953, 21, 1087-1092.

(22) Frenkel, D.; Smit, B. Understanding Molecular Simulation, 2nd ed.; Academic Press, London, UK, 2002.

(23) Binder, K.; Heermann, D. Monte Carlo Simulation in Statistical Physics, 6th ed.; Springer International Publishing, Basel, Switzerland, 2019. 